Methods A prospective review of patients undergoing endoscopic resection of neoplastic polyps in the rectosigmoid colon. Patients were tertiary referrals from experienced consultants. The polyps were considered technically challenging due to size, difficult lesion access or recurrences on previous EMR scars. Referral was made prior to surgical referral. All lesions were assessed using indigocarmine chromoendoscopy and lesions suspicious for invasive malignancy were excluded. The choice of endoscopic technique was made based on the Endoscopist's judgement of best approach for each lesion. Completeness of resection was recorded. Endoscopic follow-up was performed to assess for incomplete resection or recurrence.

Results 45 lesions were resected by ESD technique and 100 by EMR technique. For ESD procedures the median lesion size was $40 \mathrm{~mm}$ (range 20-150). 19/45 were salvage procedures post failed attempts at endoscopic resection. Endoscopic clearance was achieved at first attempt in $91 \%$ of the procedures. A further $7 \%$ were cleared in a subsequent procedure. One patient was referred for surgery for perforation and two patients went to surgery for unsuspected cancer found on histological examination of the resection specimen. At endoscopic follow-up $100 \%$ had complete clearance with no residual disease. There was one perforation requiring surgery, two microperforations (endoscopically managed), three delayed bleeds and two post polypectomy syndromes (conservatively managed) giving an overall complication rate of $18 \%$. For the EMR cohort the median lesion size was $40 \mathrm{~mm}$ (range 20-100). All procedures were primary resections. Endoscopic clearance was achieved in $90 \%$ of cases. Two patients were referred for surgery for incomplete resection. In seven patients unsuspected cancer was found, all of whom were referred for surgery. At endoscopic follow-up 95\% of cases had achieved complete clearance with no residual disease. There was seven delayed bleeds and one post polypectomy syndrome giving an overall complication rate of $8 \%$.

Conclusion Both EMR and ESD results in an excellent complete clearance rate. While ESD appears to result in fewer recurrences at follow-up it is associated with an increased complication rate compared to EMR, predominately due to the risk of perforation. However, it is effective in patients with previous failed attempts at resection. It should be considered as an option for difficult or scarred lesions where complete clearance with EMR could be difficult.

Competing interests None declared.

\section{OC-115 COMPARISON OF SCREEN DETECTED AND INTERVAL COLORECTAL CANCERS IN THE BOWEL CANCER SCREENING PROGRAMME: EXPERIENCE FROM THE NORTH EAST OF ENGLAND}

doi:10.1136/gutjnl-2012-302514a.115

\footnotetext{
${ }^{1,2,3} \mathrm{M}$ D Gill, ${ }^{* 2,3} \mathrm{M} \mathrm{G}$ Bramble, ${ }^{2,3} \mathrm{C} \mathrm{J}$ Rees, ${ }^{1,3} \mathrm{M}$ Bradburn, ${ }^{3,4} \mathrm{~T} \mathrm{~J}$ Lee, ${ }^{1,3} \mathrm{~S} \mathrm{~J}$ Mills. ${ }^{1}$ Northern Colorectal Cancer Audit Group, Northern England; ' ${ }^{2}$ niversity of Durham, Durham, UK; ${ }^{3}$ Northern Region Endoscopy Group, Northern England; ${ }^{4}$ University of Newcastle, Newcastle, UK
}

Introduction The NHS Bowel Cancer Screening Programme (BCSP) commenced screening in North East England in February 2007. It offers biennial faecal occult blood testing (FOBt) followed by colonoscopy for those with a positive FOBt. All cases of colorectal cancer (CRC) known to the MDTs in this region are registered with the Northern Colorectal Cancer Audit Group (NORCCAG) database.

Methods CRCs occurring in the screening population (aged 60-69 years) between April 2007 and March 2010 were identified from the NORCCAG database. Their screening history was obtained by cross-referencing this database with the regional BCSP database. Cases were classified into four groups: a control group (cancers diagnosed prior to first screening invite), screen detected, interval (cancers diagnosed between screening rounds after a negative FOBt) and non-uptake (patients who declined screening). Patient demographics, tumour characteristics and survival were compared between the four groups.

Results 1336 CRCs were diagnosed in the screening population. 511 (38.2\%) cancers were in the control group. 825 cancers (61.8\%) were detected in individuals who had been invited for screening. 322 $(39.0 \%)$ were screen detected, $311(37.7 \%)$ were in the non-uptake group and 192 (23.3\%) were interval cancers. All of the interval cancers followed a negative FOBt. Compared to both the control group and the interval cancer group, the proportion of males in the screen detected group was significantly higher $(73.0 \%$ vs $62.4 \%$ \& $60.4 \%, \chi^{2}=9.88, p=0.002$ and $\chi^{2}=8.77, p=0.003$ respectively). Screen detected cancers were more likely to be left sided than in the control or interval cancer groups $\left(78.6 \%\right.$ vs $70.1 \% \& 66.7 \%, \chi^{2}=7.32$, $\left.p=0.007 \& \chi^{2}=8.89, p=0.003\right)$. Significantly more Dukes A and fewer Dukes $D$ cancers were found in the screen detected group compared to the control and interval groups $(p<0.05)$. Screen detected cancers had a superior survival compared to interval cancers $\left(\chi^{2}=50.36, p<0.001\right)$ and the control group $\left(\chi^{2}=53.62, p<0.001\right)$. There was no difference in patient demographics, tumour location, stage of tumour nor survival between control and interval cancer groups.

Conclusion This is one of the first studies that provides data on the performance of the BCSP in one region since its national implementation. The FOB test is better at detecting cancers in the left colon and in men. There are significant numbers of interval cancers, which were not found to have an improved outcome compared to the non-screened population as has previously been published.

Competing interests None declared.

\section{OC-116 BOWEL SCREENING WALES-FIRST ROUND REPORT}

doi:10.1136/gutjnl-2012-302514a.116

H Heard. * Bowel Screening Wales, Llantrisant, UK

Introduction This report describes the first round of Bowel Screening Wales (BSW). It is the first overview showing the entire performance of the national bowel screening programme in Wales.

Methods The first round for BSW took place between 27 October 2008 and 24 November 2010. The Bowel Screening Information Management System obtains demographic information directly from the Welsh Demographic Service (WDS). This is the basis for the activity and outcome data presented here. This report is based on information available to BSW on the 1 August 2011

Results A total of 847773 invitation letters were issued to 412025 participants. $55.2 \%$ of participants invited returned a completed test kit within six months of invitation date. Women have a higher uptake (58.8\%) compared to men (51.5\%). Uptake around Wales varies between areas, ranging from $49 \%$ in Wrexham and Cardiff to nearly $59 \%$ in Anglesey and Bridgend. Initially, positive rates were as expected at around $0.2 \%$ for Faecal Occult Blood (FOB) kits, rising to $0.5 \%$. Positive rates for both test kits were $2.3 \%$ and $2.4 \%$ in early 2009 , rising to $3 \%$ during 2009. Around $1 \%$ of Faecal Immunochemical Test (FIT) kits were spoilt compared to $1.8 \%$ of FOB test kits. 6807 participants with a positive test result made an assessment appointment, waiting on average around 2 weeks. $82.7 \%$ attended the appointment; the majority were by phone with only seven participants attending face to face. 5594 participants were found fit for colonoscopy and offered the procedure. $89.4 \%$ of these attended with $2.8 \%$ declining and $0.2 \%$ not attending. Waiting times for colonoscopy were on average around 10 weeks. Abstract OC-116 table 1 shows the final outcome of the 5230 colonoscopy procedures. 